\title{
Aprendizaje-Servicio y construcción de materiales en la formación inicial del profesorado de Educación Física
}

\author{
Jorge ABELLÁN \\ Andrea HERNÁNDEZ-MARTÍNEZ
}

Datos de contacto:

Jorge Abellán

Universidad de Castilla-La Mancha jorge.abellan@uclm.es

Andrea Hernández-Martínez

Universidad de Castilla-La Mancha andrea.hernandez@uclm.es

Recibido: 28/10/2020

Aceptado: 24/03/2021

\section{RESUMEN}

El Aprendizaje-Servicio supone la posibilidad de aprender contenidos de los programas formativos mientras se presta un servicio a la comunidad, tratando de atender una necesidad existente en la sociedad y no cubierta. El presente trabajo presenta la realización de una experiencia de Aprendizaje-Servicio en la que participaron 34 futuros maestros de Educación Física, que debían prestar un servicio a un grupo de 14 personas con discapacidad intelectual (DI), dirigiendo sesiones de Educación Física, construyendo para ello su propio material con material reciclado. Las personas con DI no contaban con profesionales encargados de realizar sesiones de EF de manera habitual. Se evaluaron: i) creencias y opiniones sobre la utilización del material reciclado en Educación Física, con el objetivo de evaluar el aprendizaje del programa, y, la ii) opinión sobre la experiencia con dicho material y la opinión sobre el potencial educativo del Aprendizaje-Servicio, para evaluar el servicio prestado, todo ello a través de la Escala de creencias y actitudes hacia el material reciclado y la Escala de valoración de la experiencia con material reciclado. Los resultados muestran que los futuros maestros de EF mejoraron sus actitudes hacia la construcción de material reciclado y valoraron de manera positiva la implementación del programa de Aprendizaje-Servicio. Los participantes destacaron que aprendían más cuando realizaban experiencias prácticas, como es el caso del programa realizado. De manera que se concluye que la inclusión de programas de esta naturaleza puede ser adecuada para la mejora del desarrollo profesional en la formación inicial del profesorado.

PALABRAS CLAVE: formación del profesorado; aprendizaje-servicio; material reciclado; discapacidad intelectual. 


\title{
Service-Learning and homemade equipment within initial training of pre-service physical education teachers
}

\begin{abstract}
Service-Learning means the possibility to learn content from training programs while serving the community, trying to meet an existing need in society and that is not covered. This paper presents the realization of a Service-Learning experience involving 34 pre-service teachers (PTS) of Physical Education (PE), who were to serve a group of 14 people with intellectual disability (II), conducting PE sessions, building their own material with recycled material. People with II did not have professionals in charge of regular PE sessions. It evaluated: (i) beliefs and opinions on the use of recycled material in PE, with the aim of evaluating program learning, and, (ii) opinion on experience with such material and opinion on the educational potential of Service- Learning, to assess the service provided, all of this through the Scale of beliefs and attitude towards recycled material and the Scale of assesment of the experience with recycled material. The results show that PST of PE improved their attitudes towards building recycled material and positively valued the implementation of the Service-Learning program. Participants emphasized that they learned more when they were doing hands-on experiences, such as the program. Therefore, it is concluded that the inclusion of programs of this nature may be suitable for the improvement of professional development in the initial training of teachers.
\end{abstract}

KEYWORDS: teacher training; service-learning; recycled material; intellectual disability

\section{Introducción}

El Aprendizaje-Servicio (ApS) supone la posibilidad de aprender contenidos de los programas formativos mientras se presta un servicio a la comunidad, tratando de atender una necesidad existente no cubierta. Uno de sus objetivos, en la educación superior, es el de estrechar el vínculo entre el aprendizaje adquirido en la universidad y su aplicación a la realidad profesional (López-Fernández \& BenítezPorres, 2018), por ello, esta metodología tiene especial relevancia en la formación de los futuros profesionales de la educación (Bates, Drits, Allen \& McCandless, 2009), ya que, como indican García-López, Gutiérrez y Fernández-Bustos (2019), se básá en lá puestá en prácticá de conocimientos y máteriás del curri alum formál á tráves de lá párticipácion activa de los estudiántes en contextos reáles, desárrollándo trábájos utiles para la comunidad.

El ApS se ha utilizado en la formación del profesorado de Educación Física (EF), dado que el área es un marco ideal para la utilización de esta metodología debido a sus especiales características, eminentemente prácticas (Capella, Gil \& Martí, 2014). 
Esta metodología además ofrece la posibilidad de conseguir una formación teóricopráctica integral (Wilkinson, Harvey, Bloom, Joober \& Grizenko, 2013). Es por ello por lo que en los últimos años han proliferado los trabajos que combinan el ApS, la EF y la formación del profesorado (i.e. Capella-Perís, Salvador-García, Chiva-Bartoll \& Ruiz-Montero, 2020; Hernández-Martínez, Candela, Blanco \& Sánchez-Matas, 2020). Y aunque en menor medida, también existen trabajos en los que a la unión de ApS y EF se añade también el tratamiento de la diversidad (i.e. Carrington \& Saggers, 2007; Roper \& Santiago, 2014), como es el caso de la presente investigación, se entiende que por el objetivo de mejora y transformación social al que contribuye esta metodología (Hernández-Martínez et al., 2020).

En cuanto a la construcción de materiales en EF, parte del modelo de autoconstrucción de materiales, considerado como un modelo emergente en EF (Fernández-Río, Calderón, Hortigüela, Pérez-Pueyo \& Aznar, 2016), cuyo planteamiento se ha construido sobre 4 ejes: los limitados recursos del área, la existencia de presupuestos limitados, la posibilidad de crear materiales adaptables y el desarrollo de una conciencia ecológica. El modelo de autoconstrucción de materiales ha sido utilizado previamente en la formación del profesorado de EF, analizando su efecto en función de diferentes variables. En este sentido, FernándezRío y Méndez-Giménez (2013) investigaron la opinión de futuros maestros de EF y de Educación Especial tras participar en una experiencia de autoconstrucción de materiales en EF. Los resultados indicaron que la construcción de materiales permitía que los alumnos se implicaran más en el proceso de enseñanza aprendizaje. Además, los participantes observaron que los materiales autoconstruidos permitían reducir la distancia entre la teoría y la práctica.

Con estos antecedentes resulta importante diseñar, llevar a la práctica y evaluar el efecto de diferentes programas de mejora de las competencias docentes como es el caso del presente trabajo. Por ello, el objetivo fue comprobar el efecto de un programa de ApS, implementado por futuros maestros de EF, y conocer sus opiniones y actitudes hacia los materiales reciclados (evaluación del aprendizaje), y la valoración del potencial educativo del ApS (evaluación del servicio).

\section{Método}

\section{Contexto y participantes}

El alumnado de la asignatura Juegos, Ocio y Recreación $(N=34)$, perteneciente al tercer curso del Grado de Maestro en Educación Primaria de una Facultad de Educación española, desarrolló un programa de ApS junto a una asociación de personas con discapacidad intelectual (DI). Los receptores del servicio residían en un Centro de Atención a Personas con Discapacidad Intelectual impulsado por el gobierno autonómico. Dicha asociación colaboraba activamente en la realización de actividades con la Facultad de Educación y, a petición de dicha entidad, se contaba con un convenio de colaboración entre ambas desde hacía algunos años. La 
intervención que se recoge en este trabajo se desarrolló en el marco de un proyecto de innovación docente enmarcado en un Convenio de colaboración en materia de formación, innovación e investigación entre la Consejería de Educación, Cultura y Deportes de la Junta de Comunidades de Castilla-La Mancha y la Universidad de Castilla-La Mancha, en el que intervienen las cuatro Facultades de Educación.

Los participantes se dividieron entre los prestadores del servicio (futuros maestros de EF) y los receptores del servicio (adultos con DI). Sus características se detallan a continuación:

Prestadores del servicio: 34 futuros maestros de EF con una media de edad de 20.51 años $(D T=38)$. Todos los participantes estaban cursando la mención de EF. La muestra se dividía en 19 mujeres y 15 hombres. El 76.47\% de la muestra declaró haber tenido contacto previo con personas con discapacidad, bien como compañero de clase (41.17\%), amigo (26.47\%) o familiar (20.59\%). En cuanto a la experiencia previa en actividades similares, el $35.29 \%$ de los participantes había participado previamente en actividades de ApS, el 38.23\% había realizado actividad física o deporte junto a personas con discapacidad y el 50\% había construido previamente materiales reciclados en EF o deporte.

Receptores del servicio: 6 mujeres y 8 hombre con DI, cuyo rango de edad oscilaba entre 24 y 59 años. Además de la DI, algunos de ellos presentaban: discapacidad visual (una participante), discapacidad auditiva (una participante) y dificultades de movimiento (dos participantes). Estos participantes recibieron tres sesiones de EF dos horas de duración durante el ApS.

\section{Diseño de la investigación}

Se llevó a cabo un estudio cuasi-experimental con un enfoque cuantitativo, con medidas pre-post test, o post-test, en función del instrumento utilizado. El programa ApS actuó como variable independiente.

\section{Procedimiento}

Los participantes debían cursar 6 horas de un programa formativo de servicio directo (tres sesiones de dos horas de duración), en el que se les pedía que diseñaran tareas para completar 2 sesiones de EF relacionadas con los contenidos de la materia. Una sesión estuvo destinada al trabajo de los juegos de blanco y diana y otra al trabajo de los juegos inventados. En ambos casos, los futuros maestros debían diseñar y construir los materiales necesarios para llevar a cabo la actividad utilizando material reciclado. Además, en una sesión introductoria, que fue dirigida por el profesor responsable de la asignatura, se trabajaron los juegos cooperativos, otro de los contenidos propios de la asignatura. A través de estos se estableció el primer contacto entre ambos colectivos creando un clima positivo en el que primase el disfrute de la actividad.

Durante todo el proceso los participantes se distribuyeron en 12 grupos, 10 
grupos de tres personas y dos grupos de dos personas. La asignación a los grupos fue decidida por los propios estudiantes al comienzo del curso. La actividad de ApS formaba parte del proceso de evaluación continua. Todas las actividades se llevaron a cabo en un plazo de 4 meses dentro del primer cuatrimestre del curso 2019-20. Debido al carácter voluntario de la realización de los cuestionarios, enmarcado en el desarrollo académico de la asignatura, no todos los participantes cumplimentaron todos los instrumentos en cada uno de los momentos de medida. Aquellos participantes que no entregaron alguno de los cuestionarios fueron eliminados del análisis posterior. Los participantes finales de cada uno de los instrumentos se incluyen en el apartado de resultados.

El protocolo utilizado en este estudio se ajustó a las exigencias de la Declaración de Helsinki, y se encontraba aprobado dentro del protocolo del proyecto de innovación docente en el que se encuadra la presente investigación. Se obtuvieron los consentimientos informados de los participantes previamente.

\section{Diseño y validez del programa de ApS}

La Tabla 1 muestra un resumen del diseño de la experiencia. Dicho resumen está estructurado de acuerdo con las fases propuestas por Puig, Gijón, Martín y Rubio (2011), que son: diagnóstico de la realidad, desarrollo de un plan de acción, ejecución de la propuesta y obtención de resultados evaluables.

\section{Tabla 1}

Resumen programa ApS

\begin{tabular}{|c|c|c|c|}
\hline Fases & Responsables & Objetivos & Resultados \\
\hline $\begin{array}{l}\text { Diagnóstico de } \\
\text { la realidad }\end{array}$ & $\begin{array}{l}\text { Educador de la } \\
\text { asociación }\end{array}$ & $\begin{array}{l}\text { Dar a conocer la ausencia } \\
\text { de profesionales de EF en } \\
\text { su asociación por falta de } \\
\text { recursos y la necesidad de } \\
\text { realizar EF para la mejora } \\
\text { de la calidad de vida de sus } \\
\text { usuarios. }\end{array}$ & $\begin{array}{l}\text { El profesor de la asignatura } \\
\text { se compromete a realizar un } \\
\text { servicio directo, dentro de } \\
\text { un proyecto de innovación } \\
\text { docente de ApS concedido a } \\
\text { la Facultad. }\end{array}$ \\
\hline
\end{tabular}




\begin{tabular}{llll}
\hline $\begin{array}{l}\text { Desarrollo de } \\
\text { un plan de } \\
\text { acción }\end{array}$ & Profesor de la & $\begin{array}{l}\text { Diseñar un plan de trabajo } \\
\text { e incluirlo dentro de la } \\
\text { programación, en la }\end{array}$ & $\begin{array}{l}\text { El programa ApS se incluye } \\
\text { en la guía docente de la } \\
\text { asignatura, asignándole el }\end{array}$ \\
& & modalidad de evaluación & $20 \%$ de la nota final. \\
continua. & Se indica que se deben \\
Evaluar las creencias & trabajar los siguientes \\
previas respecto a la & contenidos de la asignatura: \\
& utilización de material & juegos cooperativos, juegos \\
reciclado en EF (Pre-test) & de blanco y diana y \\
& & & construcción de material \\
& & & \\
& &
\end{tabular}

\begin{tabular}{|c|c|c|c|}
\hline $\begin{array}{l}\text { Ejecución de la } \\
\text { propuesta }\end{array}$ & $\begin{array}{l}\text { Alumnado de Juegos, } \\
\text { ocio y recreación }\end{array}$ & $\begin{array}{l}\text { Participar activamente en } \\
\text { una sesión de juegos } \\
\text { cooperativos, que se utiliza } \\
\text { para que ambos colectivos } \\
\text { se conozcan (diagnóstico } \\
\text { de la realidad para el } \\
\text { alumnado). } \\
\text { Diseñar } 2 \text { sesiones de EF y } \\
\text { construir el material } \\
\text { necesario para trabajar los } \\
\text { contenidos con dicho } \\
\text { material. }\end{array}$ & $\begin{array}{l}\text { Se diseñan } 24 \text { juegos, en dos } \\
\text { sesiones, con sus respectivos } \\
\text { materiales, creados a tal } \\
\text { efecto. }\end{array}$ \\
\hline
\end{tabular}

\begin{tabular}{l|l|l|l|}
\hline $\begin{array}{l}\text { Obtención de } \\
\text { resultados } \\
\text { evaluables }\end{array}$ & $\begin{array}{l}\text { Profesor de la } \\
\text { facultad }\end{array}$ & $\begin{array}{l}\text { Evaluar el impacto del ApS } \\
\text { en las creencias respecto al } \\
\text { material reciclado y la } \\
\text { opinión del alumnado }\end{array}$ & $\begin{array}{l}\text { Se obtienen los resultados } \\
\text { presente trabajo. }\end{array}$ \\
& & $\begin{array}{l}\text { respecto al potencial } \\
\text { educativo del ApS (Post- } \\
\text { test). }\end{array}$ \\
\hline
\end{tabular}

De manera específica, la aplicación del programa ApS pretendía la consecución de las siguientes competencias de la titulación del Grado de Maestro en Educación Primaria, incluidas en la guía docente de la asignatura Juegos, ocio y recreación (https://guiae.uclm.es/vistaGuia/307/46340):

- CG02: Diseñar, planificar y evaluar procesos de enseñanza y aprendizaje, tanto individualmente como en colaboración con otros docentes y profesionales del centro.

- CG04: Diseñar y regular espacios de aprendizaje en contextos de diversidad y que atiendan a la igualdad de género, a la equidad y al respeto a los derechos humanos que conformen los valores de la formación ciudadana.

La actuación cumple con los requisitos para ser considerado como ApS, de acuerdo con Capella et al. (2014), ya que durante la ejecución de la propuesta existe 
un papel del profesor como guía, mientras que son los alumnos los que planifican y dirigen. Además, los receptores del servicio son un colectivo en riesgo de exclusión, y se trabajaron tanto la educación en valores (atención a la diversidad) como los contenidos específicos de la asignatura (juegos de blanco y diana y autoconstrucción de materiales).

\section{Instrumentos}

Los instrumentos utilizados, que se detallan a continuación, permitieron conocer aspectos relacionados con el aprendizaje (construcción de material en EF), y aspectos concernientes al servicio prestado (valoración del potencial educativo $\operatorname{del} \mathrm{ApS})$.

Construcción de materiales alternativos para EF: para conocer las actitudes y opiniones de los futuros maestros de EF sobre su proceso de construcción de material alternativo, utilizado durante la experiencia, se utilizó el cuestionario elaborado por Méndez-Giménez y Fernández-Río (2012), compuesto por 40 ítems distribuidos en dos escalas: la Escala de creencias y actitudes hacia el material reciclado (ECAMA) y la Escala de valoración de la experiencia con material reciclado (EVEMA) (Méndez-Giménez \& Fernández-Río, 2012). La ECAMA está compuesta por 20 ítems repartidos en 4 dimensiones: material reciclado como herramienta metodológica (4 ítems), como estrategia para trabajar la interdisciplinariedad (6 ítems), en relación con la educación en valores (4 ítems) y como herramienta de evaluación ( 6 ítems). Por otro lado, la EVEMA está compuesta por 20 ítems que evalúan el grado de utilidad, esfuerzo, motivación, satisfacción y compromiso vivido por cada participante. En ambas escalas los participantes debían valorar su grado de acuerdo con las afirmaciones utilizando una escala tipo Likert de 5 puntos (de 1 $=$ muy en desacuerdo a $5=$ muy de acuerdo). La primera escala (ECAMA) fue aplicada antes y después de la realización de la ApS, mientras la segunda (EVEMA) solo se implementó en el post-test. La ECAMA mostró un Alfa de Cronbach de 92 mientras que la EVEMA mostró una fiabilidad de .95.

Valoración del potencial educativo de la experiencia de ApS: con el objetivo de conocer las opiniones y actitudes de los futuros maestros de EF después de la realización del ApS, y su valoración del potencial educativo de esta metodología, se les administró el Cuestionario sobre la opinión y actitud hacia el ApS elaborado por López-Fernández y Benítez-Porres (2018), a partir del trabajo de Prentice y Robinson (2010). Este cuestionario está compuesto por 13 ítems, que los participantes debían valorar en una escala tipo Likert de 4 puntos (de $1=$ muy en desacuerdo a 4 = muy de acuerdo). La escala mostró un Alfa de Cronbach de .81.

\section{Análisis estadístico}

Se tomaron los valores medios de las escalas y dimensiones como variables dependientes y el programa ApS como variable independiente. Se realizaron 
pruebas no paramétricas tras comprobar que los datos no seguían una distribución normal. Para comparar el efecto de la intervención se realizó la comparación por pares (pre-test vs. post-test) utilizando la prueba de los rangos con signo de Wilcoxon.

\section{Resultados}

Los resultados se presentan separados en función de las variables analizadas, relacionadas por un lado con el aprendizaje (construcción de materiales alternativos para EF) y, por el otro lado, con el servicio (valoración del potencial educativo del ApS).

\section{Construcción de materiales alternativos para EF}

Las valoraciones de los participantes sobre su experiencia con la construcción de material reciclado en EF se presentan en las Tabla 2 (ECAMA) y Tabla 3 (EVEMA).

\section{Tabla 2}

Diferencias en la administración de la ECAMA $(n=25)$.

\begin{tabular}{|c|c|c|}
\hline & Pre-test & Post-test \\
\hline $\begin{array}{l}\text { Herramienta } \\
\text { metodológica* }\end{array}$ & $3.97 \pm 0.45$ & $4.22 \pm 0.45$ \\
\hline Interdisciplinariedad* & $3.99 \pm 0.51$ & $4.22 \pm 0.45$ \\
\hline Educación en valores & $4.44 \pm 0.52$ & $4.52 \pm 0.42$ \\
\hline Evaluación & $3.77 \pm 0.61$ & $3.93 \pm 0.74$ \\
\hline
\end{tabular}

Nota. $(*)$ Indica diferencias significativas entre grupos para $p<.05$

Como puede observarse en la Tabla 2, todas las dimensiones mejoran trás la realización del ApS. Estas mejoras son significativas a nivel estadístico en las dimensiones Herramienta metodológica $(Z=-1.981 ; p=.048)$ e Interdisplinariedad $(Z=-2.026 ; p=.043)$. En ambos casos el efecto de la realización de la experiencia mejoró la percepción de los participantes sobre la utilización de la construcción de material en EF como herramienta metodológica y como elemento para trabajar la interdisciplinariedad. 


\section{Tabla 3}

\section{Resultados en la administración de la EVEMA $(n=24)$.}

$\mathrm{M} \pm \mathrm{DT}$

1. Me ha resultado fácil encontrar la materia prima para elaborarlos

$4.04 \pm .69$

2. Me ha supuesto esfuerzo construirlos

3. Me han permitido conocer contenidos y actividades nuevas

4. Me han permitido mejorar mis habilidades motrices

5. Los he encontrado útiles para abordar esta asignatura

6. Han restado tiempo para abordar los contenidos de esta asignatura

7. Han supuesto un beneficio significativo para mí como alumno

8. Me han parecido rentables considerando el gasto económico y su funcionalidad

$4.21 \pm .88$

9. Estoy satisfecho con la experiencia de utilizarlos para aprender esta asignatura

10. Espero poder emplearlos cuando sea profesor

11. Han contribuido mucho en mi aprendizaje práctico de la asignatura

12. Me han permitido mostrar capacidades poco evaluadas en Educación Física

13. Han despertado mi interés por la asignatura

14. Me han motivado para aprender los contenidos de la asignatura

15. Me han permitido acceder a un conocimiento más significativo, más motivador

16. Han encajado muy bien con mi forma de aprender

17. Han aumentado mi compromiso con la asignatura

18. Han propiciado que trabaje en grupo, aumentado interacción con compañeros

19. Me siento muy orgulloso de los materiales que he construido

El ítem menos valorado ha sido el 6 "Hán restado tiempo para abordar los contenidos de esta ásignáturá" (3.21£1.1), seguido del 4 "Me han permitido mejorar 


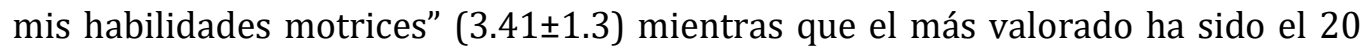
"Ahorá valoro más los materiales que yo he construido y los de los demás"

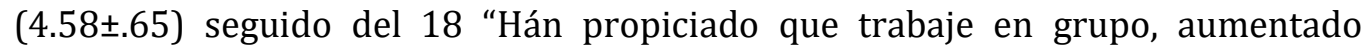
interacción con compáñeros” (4.54t.66).

\section{Valoración del potencial educativo del ApS}

Las opiniones sobre el potencial educativo del ApS se presentan en la Tabla 3.

\section{Tabla 3}

Resultados de la opinión de los participantes sobre el potencial educativo del ApS ( $n=$ 33).

\section{$\mathrm{M} \pm \mathrm{DT}$}

1. Aprendo mejor la asignatura cuando se relaciona con situaciones

$3.88 \pm .33$ escolares reales

2. Las experiencias de ApS mejoraron la relación con mis compañeros de

$3.21 \pm .65$ clase

3. Gracias a las experiencias de ApS comprendo mejor mi rol como docente.

4. En las experiencias de ApS aprendí algo nuevo sobre cómo trabajar como docente

5. Habría aprendido más si el tiempo invertido en el ApS se hubieran dado clases normales en el aula

6. Después de las experiencias de ApS tengo más claro lo que quiero hacer en mi vida profesional

7. Las experiencias de ApS influyeron en mi deseo de seguir aprendiendo

$3.39 \pm .66$

$3.24 \pm .79$

$1.91 \pm 1.01$

$3.12 \pm .74$

$3.18 \pm .77$

8. Las experiencias de ApS me ayudaron a saber cómo aplicar la teoría a

$3.18 \pm .72$ la práctica real

9. Me gustaría que mis otras asignaturas incluyeran experiencias de ApS

$3.24 \pm .66$

10. Las clases desarrolladas dentro del aula (teoría) contribuyeron de manera significativa a mi aprendizaje

11. Las prácticas de ApS contribuyeron de manera significativa a mi aprendizaje

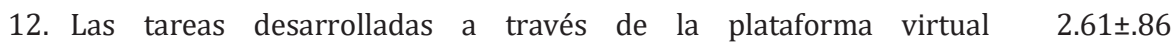
contribuyeron de manera significativa a mi aprendizaje

13. Las prácticas desarrolladas en el pabellón contribuyeron de manera significativa a mi aprendizaje 
El ítem menos valorado ha sido el 5 "Hábríá aprendido más si el tiempo invertido en el ApS se hubieran dado clases normales en el áulá” (1.91ะ1.01), seguido del 12 "Lás tareas desarrolladas a través de la plataforma virtual contribuyeron de manera

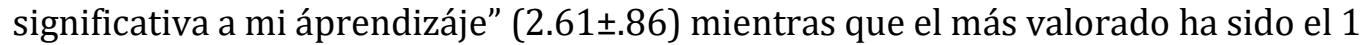
"Aprendo mejor la asignatura cuando se relaciona con situaciones escolares reáles" (3.88土.33) seguido del 13 “Lás prácticas desarrolladas en el pabellón contribuyeron

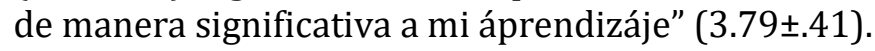

\section{Discusión}

El objetivo de la presente investigación fue comprobar el efecto de un programa de ApS en la formación del profesorado de EF, relacionado principalmente con las competencias de la titulación del Grado de Maestro en Educación Primaria, citados en el apartado de diseño y validez del programa. Para obtener una visión amplia del impacto del programa se analizó, por un lado el aprendizaje, a través de la construcción de material alternativo en EF, y por otro lado el servicio prestado, valorando el potencial educativo del ApS.

Se puede considerar que la experiencia $\mathrm{ApS}$ ha resultado positiva para los prestadores del servicio, ya que la valoración del ApS y de la utilización del material reciclado han mostrado valores muy positivos. Es reseñable que el ítem más valorado en la escala de opinión del potencial educativo del ApS (López-Fernández \& Benítez-Porres, 2018) haya sido el 1 "Aprendo mejor la asignatura cuando se relaciona con situaciones escolares reáles" y el ítem menos valorado haya sido el 5 "Hábríá aprendido más si el tiempo invertido en el ApS se hubieran dado clases normales en el aulá". En este sentido, los participantes manifestaron aprender mejor las diferentes asignaturas cuando se relacionan con situaciones escolares reales, como ha sido el caso de esta experiencia, porque el aprendizaje es altamente significativo, además de valorar positivamente las prácticas desarrolladas en el pabellón por su contribución al aprendizaje y su conexión con la realidad.

También la opinión de la utilización del material reciclado (Méndez-Giménez \& Fernández-Río, 2012) presenta resultados similares, ya que el ítem menos valorado ha sido el 6 "Hán restado tiempo para abordar los contenidos de esta ásignáturá". Parece por tanto que, el empleo de la metodología ApS y la construcción y empleo en acciones de práctica real del material reciclado, han conseguido, al menos inicialmente, tender un puente entre los contenidos aprendidos en el contexto universitario y el desempeño docente real.

Esta idea de acortar las distancias entre el conocimiento teórico y práctico de los futuros docentes ya había sido manifestada por Fernández-Río y MéndezGiménez (2013). En dicho trabajo los participantes mostraron que, el hecho de haber tenido que construir materiales didácticos de EF con material reciclado, había contribuido al aprendizaje práctico de los contenidos (Fernández-Río \& MéndezGiménez, 2013). Al igual que en el presente trabajo, la escasa puntuación del ítem referido a que dicha construcción habría restado tiempo al desarrollo de otros 
contenidos, indica que los participantes habrían percibido que los materiales reciclados se integraban en su formación, añadiendo un componente práctico, en línea con lo señalado por Pérez-Gómez (2010) quién indicó que la formación de los futuros docentes no debería estar tan centrada en aspectos teóricos. En esta línea se encuentran también los resultados de la valoración del potencial educativo del ApS.

En consonancia con lo indicado previamente por López-Fernández y BenítezPorres (2018), la opinión generalizada del alumnado es que han aprendido más dedicando el tiempo a realizar experiencias de ApS que a clases "normáles". La ocasión de construir materiales de acuerdo con las características específicas del alumnado al que va destinado (Fernández-Río, Hortigüela \& Pérez-Pueyo, 2018), y la posibilidad de hibridar este modelo con otras estrategias dentro de la EF (Fernández-Río et al., 2016), justifican su inclusión dentro del programa ApS analizado en el presente trabajo.

En referencia a las actitudes hacia la utilización de material reciclado de los participantes, mejoraron significativamente tras la realización del programa en cuanto a la valoración de dicho material como herramienta metodológica y como elemento para trabajar la interdisciplinariedad. No consideran que hayan perdido el tiempo en la asignatura ante el hecho de construir materiales, al contrario, valoran más el material autoconstruido y han mejorado la relación con los compañeros como consecuencia de trabajar en grupo. A tal respecto, MéndezGiménez y Fernández-Río (2013) mostraron además que, las intervenciones utilizando la construcción de materiales, aumentaban sus creencias y expectativas sobre su uso como futuros profesionales de la EF.

La mejora en el apartado Herramienta metodológica es congruente con el programa específico analizado en el presente trabajo, ya que en dicho apartado se incluyen dos ítems directamente relacionados con la atención a la diversidad. También en el trabajo de Fernández-Río y Méndez-Giménez (2013) los participantes pertenecientes a la especialidad de Educación Especial habían percibido que, la posibilidad de construir materiales en EF, podría ser una herramienta para adaptarlos a las necesidades educativas especiales del alumnado con discapacidad. En cuanto a la mejora en el apartado de interdisciplinariedad, los resultados están de acuerdo con el trabajo de Méndez-Giménez et al. (2016). Los participantes de esta investigación percibieron que la construcción de material en EF podía servir para trabajar aspectos comunes con otras materias e indicaron la posibilidad de investigar cómo desarrollar estos proyectos insterdisciplinares y sus posibles efectos en el desarrollo de competencias comunes.

\section{Conclusiones}

Teniendo en cuenta los objetivos propuestos y la naturaleza experiencial del presente trabajo, se puede afirmar que la realización de un programa de ApS en EF con construcción de materiales reciclados tiene un efecto positivo en la actitud hacia 
el material reciclado en EF y la opinión del potencial educativo del ApS en la formación inicial del profesorado de EF.

Se puede decir que esta experiencia contribuye a la consecución de las competencias de la titulación del Grado de Maestro en Educación Primaria en cuanto al diseño, planificación y evaluación, tanto de procesos de enseñanza y aprendizaje, de manera individual o en colaboración con otros profesionales, como en su respuesta a contextos de diversidad en los que se atienda a la equidad y al respeto de los derechos humanos.

A la luz de estos resultados se sugiere la necesidad de incorporar este tipo de programas en los estudios habilitantes de la profesión de maestro con el objetivo de mejorar su futuro desarrollo profesional en el campo de conocimiento de la EF.

El presente trabajo expone también una serie de limitaciones, que se enumeran acompañadas de posibles prospectivas de investigación que ayuden a avanzar en la formación del profesorado de EF. En primer lugar, la escasez de la muestra y la ausencia de grupo control hacen que los resultados deban ser tomados con cautela, constantando el efecto de programas similares con muestras más amplias y utilizando diseños que incluyan un grupo control. Por lo tanto, la naturaleza de experiencia didáctica del presente trabajo limita la posibilidad de extrapolar los resultados, al utilizar un grupo clase natural y describir una intervención puntual. En segundo lugar, se aconseja incorporar en futuras investigaciones una perspectiva mixta, completando los datos cuantitativos obtenidos por medio de escalas y cuestionarios validados, con datos cualitativos que permitan ahondar en el efecto de programas similares sobre la formación del profesorado de EF.

\section{Referencias}

Bates, A. K., Drits, D., Allen, C., y McCandless, P. (2009). Service learning as an instructional strategy for the preparation of teachers. The Journal of Effective Teaching, 9, 5-23.

Capella, C., Gil, J., y Martí, M. (2014). La metodología del aprendizaje-servicio en la educación física. Apunts. Educación Física y Deportes, 116(2), 33-43. https://doi.org/10.5672/apunts.20140983.es.(2014/2).116.03

Capella-Perís, C., Salvador-García, C., Chiva-Bartoll, Ó., y Ruiz-Montero, P. J. (2020). Alcance del aprendizaje-servicio en la formación inicial docente de educación física: una aproximación metodológica mixta. Retos: nuevas tendencias en educación física, deporte y recreación, 37, 465-472.

Carrington, S., y Saggers, B. (2008). Service-learning informing the development of an inclusive ethical framework for beginning teachers. Teaching and Teacher Education 24, 795-806. https://doi.org/10.1016/j.tate.2007.09.006

Fernández-Río, J., y Méndez-Giménez, A. (2013). Articulando conocimiento teórico y práctica educativa. Análisis de los efectos del material autoconstruido en las creencias de futuros docentes. Infancia y Aprendizaje, 36(1), 61-75. 
https://doi.org/10.1174/021037013804826528

Fernández-Río, J., Calderón, A., Hortigüela, D., Pérez-Pueyo, A., y Aznar, M. (2016). Modelos Pedagógicos en Educación Física: Consideraciones teórico-prácticas para docentes. Revista Española de Educación Física y Deportes, 413, 55-75.

Fernandez-Río, J., Hortigüela, D., y Pérez-Pueyo, Á. (2018). Revisando los modelos pedagógicos en educación física. Ideas clave para incorporarlos al aula. Revista Española de Educación Física y Deportes, 423, 57-80.

García-López, L. M., Gutiérrez, D., y Fernández-Bustos, J. G. (2019). Emprendimiento docente en lá tránsicion de Educácion Primáriá á Educácion Secundáriá: uná propuestá desde lá Educácion deportiva y el Aprendizajeservicio. Contextos Educativos, 24, 113-121.

Hernández-Martínez, A., Candela, P., Blanco, M. M., y Sánchez-Matas, Y. (2020). El Aprendizaje-servicio en la Universidad de Castilla-La Mancha: una apuesta para llevar la transdisciplinariedad a las aulas. Póster presentado en las XVIII Jornadas de Redes de Investigación en Docencia Universitaria y el IV Workshop Internacional de Innovación en Enseñanza Superior y TIC, REDESINNOVAESTIC, celebrado en Alicante, 4-11 de junio de 2020.

López-Fernández, I., y Benítez-Porres, J. (2018). El Aprendizaje Servicio en la Universidad: una experiencia en el marco de una asignatura del Grado en Educación Primaria. REDU. Revista de Docencia Universitaria, 16(2), 195-210. https://doi.org/10.4995/redu.2018.9127

Méndez-Giménez, A., y Fernández-Río, J. (2012). Efecto de los estresores académicos en las creencias del alumnado de magisterio sobre el material reciclado. Revista Electrónica Interuniversitaria de Formación del Profesorado, 15(3), 161-171.

Méndez-Giménez, A., y Fernández-Río, J. (2013). El aprendizaje cooperativo en la formación del profesorado: una experiencia basada en autoconstrucción de materiales e invención de juegos. Revista Española de Educación Física y Deportes, 400, 55-75.

Pérez-Gómez, A. I. (2010). La naturaleza del conocimiento práctico y sus implicaciones en la formación de los docentes. Infancia y Aprendizaje, 33(2), 171-177. https://doi.org/10.1174/021037010791114652

Prentice, M., y Robinson, G. (2010). Improving Student Learning Outcomes with Service Learning. American Association of Community Colleges (NJ1). Recuperado de https://eric.ed.gov/?id=ED535904

Puig, J. M., Gijón, M., Martín, X., y Rubio, L. (2011). Aprendizaje-servicio y educación para la ciudadanía. Revista de Educación, 298, 45-67.

Roper, E. A., y Santiago, J. A. (2014). Influence of service-learning on kinesiology students' attitudes toward P-12 students with disabilities. Adapted Physical Activity Quarterly, 31, 162-180. https://doi.org/10.1123/apaq.2013-0086 
Wilkinson, S., Harvey, W. J., Bloom, G. A., Joober, R., y Grizenko, N. (2013). Student teacher experiences in a service-learning project for children with attentiondeficit hyperactivity disorder. Physical Education \& Sport Pedagogy, 18(5), 475-491. https://doi.org/10.1080/17408989.2012.690385 
162 ISSN 0213-8646 | E-ISSN 2530-3791 • Revista Interuniversitaria de Formación del Profesorado, 96 (35.1) (2021), 147162 